\title{
In vitro Assessment of Coronal
Discoloration from Endodontic Sealers \\ In vitro Assessment of Coronal
Discoloration from Endodontic Sealers
}

\author{
Makdad Chakmakchi \\ BDS.MSc,PhD (Asst.Prof) \\ Chris Rahiotis \\ MSc.,PhD (Lec.) \\ Nikolas P. Kerezoudis \\ MSc,PhD (Asst.Prof) \\ Afrodite Kakaboura \\ MSc.,PhD (Prof.)
}

Department of conservative Dentistry College of Dentistry, University of Mosul

Department of operative Dentistry School of Dentistry, University of Athens, Athens, Greece

Department of Endodontic Dentistry School of Dentistry, University of Athens, Athens, Greece

Department of operative Dentistry School of Dentistry, University of Athens, Athens, Greece

\begin{abstract}
الخلاصة

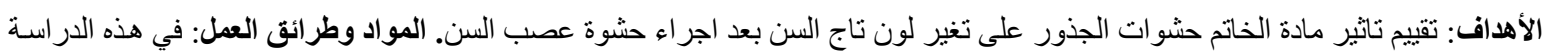

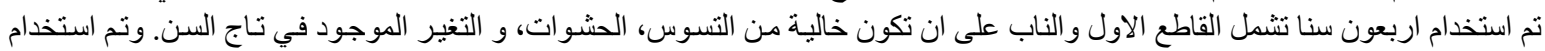

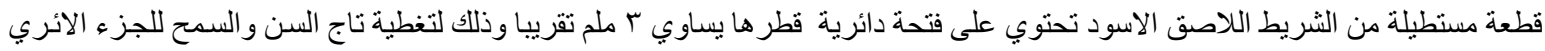

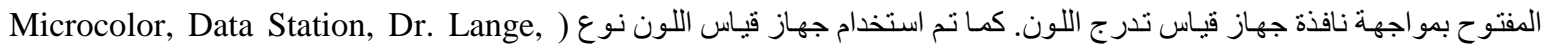

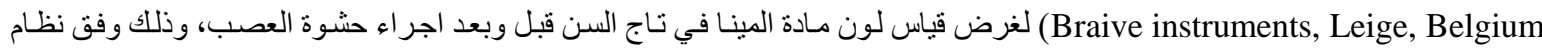
(CIE-L*a*b*)

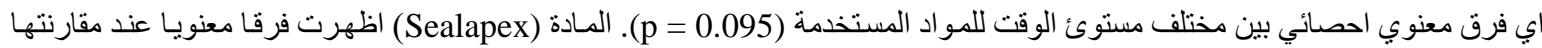

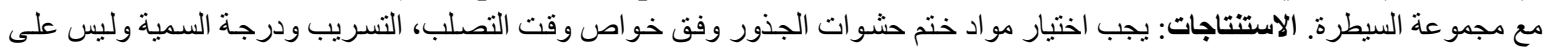
اساس مقدار الثاثير في لون السن.
\end{abstract}

\begin{abstract}
Aims: to evaluate the effect of different endodontic sealers on the coronal discoloration of the tooth part. Materials and methods: Forty intact human upper incisors and canines free of caries, restorations and coronal discoloration were used in the study. Black rectangular pieces of adhesive tape, with a round opening of $3 \mathrm{~mm}$ in diameter was cut to match the size of colorimeter window, the tape was applied to the middle third of the buccul surface of the tooth to facilitate a means of enamel surface standardization for analysis and was further secured to the teeth crowns with cyanoacrylate glue. A colorimeter (Microcolor, Data Station, DeLange, Braive instruments, Leige, Belgium) according to the CIE-L*a*b* color system was used to evaluate the color of enamel in the coronal part before and after instrumentation and obturation of the teeth, employing a repeated measures design $(n=5)$ per sample. Results: There was no statistically significant difference among the levels of time of different materials $(\mathrm{p}=0.095)$. Sealapex was presented significant differences against control group. Conclusion: The selection of endodontic sealers should be based mainly on other criteria (setting time, microleakage and toxicity) and no on the potent ional staining.
\end{abstract}

Key words: Coronal discoloration, Endodontic sealers.

Chakmakchi M. Rahiotis C., Kerezoudis N., Kakaboura A. In vitro Assessment of Coronal Discoloration from Endodontic Sealers. Al-Rafidain Dent J. 2020 ;20 (2):177- 182.

DOI: 10.33899/rden.2020.166457_ @2020, College of Dentistry, University of Mosul

Received: 1/10/2013 Sent to Referees: 15/10/2013 Accepted for Publication: 6/11/2013

This is an open access article under theCCBY4.0license (http://creativecommons.org/licenses/by/4.0/). 


\section{INTRODUCTION}

Tooth discolouration following endodontic treatment is a common aesthetic problem in clinical dentistry, especially when the anterior dentition is indicated that strongly affect the appearance of the aesthetic zone. ${ }^{(1)}$ Few times iatrogenic induced discoloration result from dental procedures. The major causes of discolouration are haemorrhage into the pulp cavity, decomposition of necrotic pulp tissue, tissue remnants after endodontic treatment, discoloration of pulp canal sealers and filling material, root resorption and aging. $(2,3)$

The reports on the discoloration potential of endodontic materials provided in dental literature are often incomplete or even contradictory, due to the lack of systematic research in this field. ${ }^{(3-6)}$ Several studies have evaluated the discoloration potential of sealers and the patterns and changes over the time. ${ }^{(7)}$ Despite the relatively extensive evidence available on potential staining of endodontic sealers, a considerable variability can be seen among results of same materials, probably deriving from the multiplicity of test configuration, as well as the assumptions and approximation integrated in experimental methodologies. The aim of this study was to investigate the coronal discoloration induced by three different types of endodontic sealers. The research hypothesis suggest that there is no difference in the coronal discoloration induced by these sealers at different time level.

\section{MATERIALS AND METHODS}

Forty intact human upper incisors and canines free of caries, restorations and coronal discoloration were used in the study. The teeth were extracted for periodontal reason and stored in distilled water prior to the study, the teeth were thoroughly cleaned, polished with slurry of pumice and water in rubber prophylaxis cup at low speed. On black rectangular pieces of adhesive tape, a round opening of $3 \mathrm{~mm}$ in diameter was cut to match the size of colorimeter window. The tape was applied to the middle third of the buccul surface of the tooth to facilitate a means of enamel surface standardization for analysis and was further secured to the teeth crowns with cyanoacrylate glue. The teeth were codenumbered for identification purposes, and the exposed enamel windows were evaluated by means of a colorimeter (Figure 1) (Micro color, Data Station, Dr. Lange, Braive instruments, Leige, Belgium) according to the CIE-L*a*b* color system employing a repeated measures design $(n=5)$ per sample. The CIE colour L* parameter corresponds to the value or degree of lightness in the Munsell system, whereas the $\mathrm{a}^{*}$, and $\mathrm{b}^{*}$ co-ordinates designate positions on $\mathrm{red} /$ green $\quad\left(+\mathrm{a}^{*}=\mathrm{red}, \quad-\mathrm{a}^{*}=\right.$ green $) \quad$ and yellow/blue $\left(+b^{*}=\right.$ yellow, $-b^{*}=$ blue $)$ axes. 


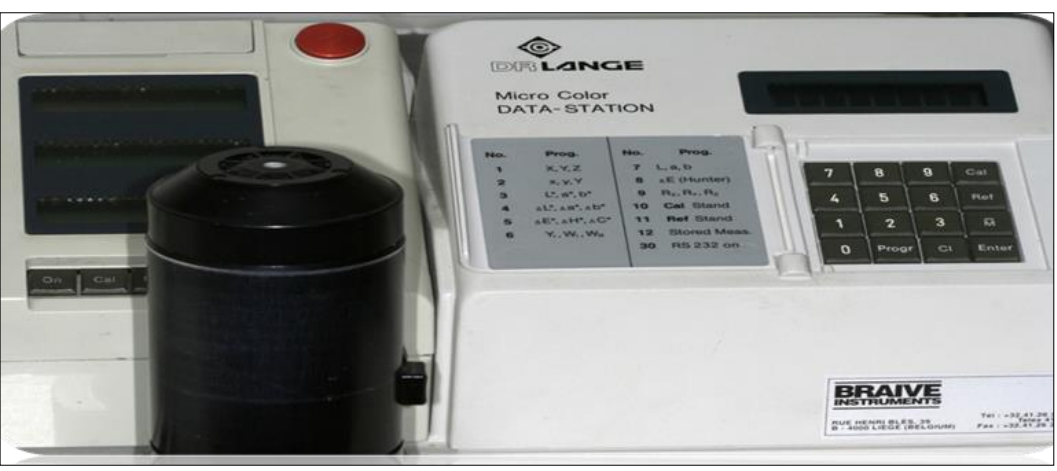

Figure (1): Dr. Lange Micro Color: color measuring device.

Afterwards, access and step-back laterally condensed with a fine finger spreader instrumentation with K-files (Maillefer, (Kerr), then accessory cones were added as Detrey/Denstply, Zurich, Switzerland) were before. The gutta-percha was removed at the performed on all teeth using $5 \% \mathrm{NaOCl}$ as an irrigant and canals were dried thoroughly with paper points and temporization was performed with a dry cotton pellet and cavit. The teeth were randomly classified into four groups ( $\mathrm{n}=10$ per group). The teeth were kept in wet condition. After 1-week storage, the root canals of the teeth were filled with a sealer and gattapercha points employing the lateral condensation technique. The sealer used were: Sealapex (Sybron-Kerr, Romulus, MI, USA) in the first group, Roth's 801 (Roth's International, Chicago, IL, USA) in the second group and AH-26 (DeTrey/Dentsply, Zurich, Switzerland) in the third group. In the fourth, no sealer was used (control group). The sealers were used according to the manufacturers` recommendations. The sealers were placed in the root canals by coating, inserting and spinning a lentalo file counter clockwise. Sealers were also placed on master cones; level of cement-enamel junction. After that, all teeth were filled with sticky wax and stored for two months at $100 \% \mathrm{RH}$ and $37{ }^{\circ} \mathrm{C}$.

The colour measurements were performed, before any preparation (baseline/t0), after the instrumentation (t1), after the obturation (t2), and following after one month (t3) and two months storage after-obturation (t4). The color difference $\Delta \mathrm{E}$ for each sample was calculated from the baseline measurement and any time interval using the following equation: $\Delta \mathrm{E}=\left(\Delta \mathrm{L}^{* 2}+\Delta \mathrm{a}^{2}+\Delta \mathrm{b}^{* 2}\right)^{1 / 2}$ The $\Delta \mathrm{E}$ values of the four sealing conditions to the four time intervals, were statistically analyzed using two-way ANOVA (a:0.05) employing sealer type and storage condition as independent variables.

\section{RESULTS}

Table 1 and Figure 2 presents the results of $\Delta \mathrm{E}$ values among the different sealers and time intervals. 
Table (1): the $\Delta \mathrm{E}$ difference sealer with respect to time condition used.

\begin{tabular}{ccccc}
\hline $\begin{array}{c}\text { color difference } \\
(\text { Time })\end{array}$ & Sealapex & Roth & AH-26 & Control \\
\hline$\Delta \mathbf{E}(\mathbf{t} 1-\mathbf{t 0})$ & $2.33 \pm 0.16$ & $2.09 \pm 0.10$ & $2.07 \pm 0.08$ & $1.99 \pm 0.11$ \\
$\Delta \mathbf{E}(\mathbf{t} 2-\mathbf{t 0})$ & $2.73 \pm 0.12$ & $2.26 \pm 0.13$ & $2.08 \pm 0.07$ & $2.08 \pm 0.09$ \\
$\Delta \mathbf{E}(\mathbf{t} 3-\mathbf{t 0})$ & $3.16 \pm 0.05$ & $2.52 \pm 0.1$ & $2.26 \pm 0.11$ & $2.17 \pm 0.13$ \\
$\Delta \mathbf{E}(\mathbf{t} 4-\mathbf{t 0})$ & $3.17 \pm 0.15$ & $2.61 \pm 0.08$ & $2.55 \pm 0.12$ & $2.30 \pm 0.13$ \\
& & & & \\
\hline
\end{tabular}

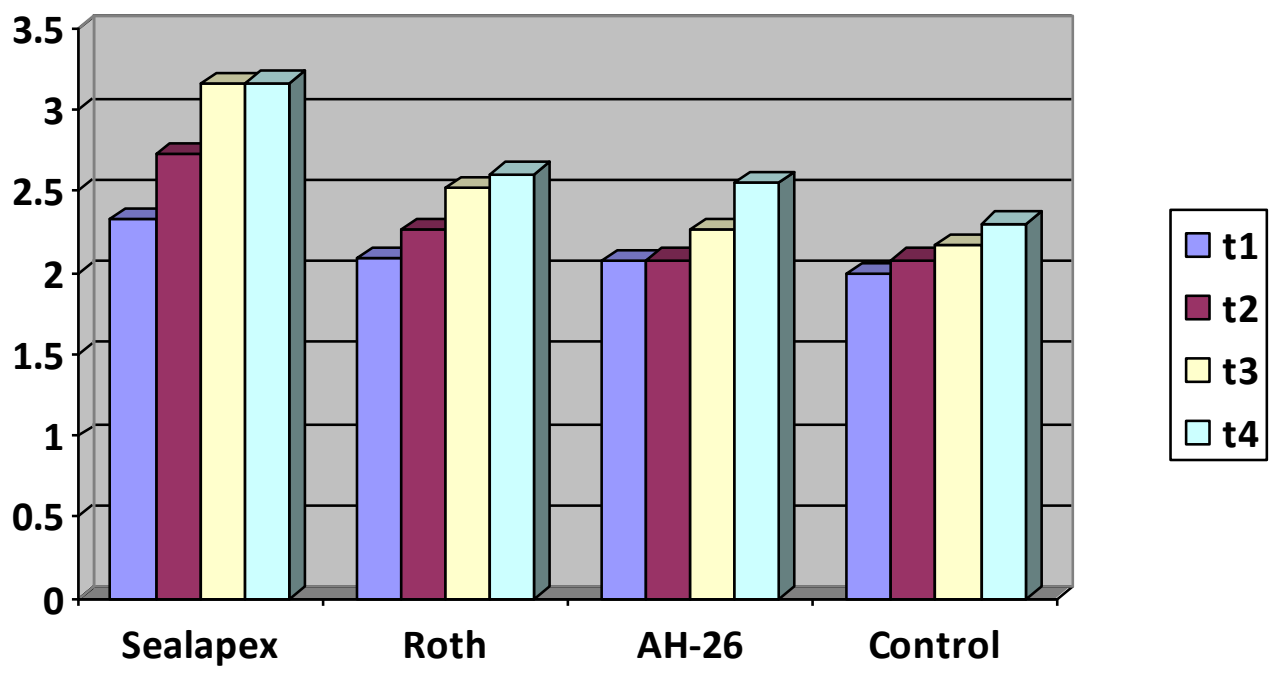

Figure (2): Histogram showing the $\Delta \mathrm{E}$ difference of a different sealers with respect to time condition used

Two-way ANOVA revealed no statistically significant difference among the different levels of time $(\mathrm{p}=0.095)$. Sealapex was presented significant differences against control group.
DISCUSSION

Although, it has been considered that differences exceeding 2 units ( $\Delta \mathrm{E}$ values) indicate colour change, previous study has indicated that the proposed acceptance limit for colour matching to 3.7 units, beyond which the differences are clinically visible. ${ }^{(8)}$ In the 
present study all the colour difference were not found to exceed this threshold value for clinical detection. An interesting find of the current study was that the larger differences observed for the baseline-instrumentation ( $\mathrm{t} 1$ ) interval that may be explained by the change in optical properties due to the access cavity preparation.

The present study revealed no significant difference between the endodontic sealer regards to their ability to discolour teeth with time. Thus, the research hypothesis had to be accepted.

The findings of this study are contradictory to the others investigations by Van der Burgt et al and Parsons et al. ${ }^{(4,6)}$. On the aforementioned studies the sealer was placed into pulp champers and not into root canal, resulting in the chamber being filed with the sealer. In the current study we tried to simulate the real clinical conditions during the endodontic treatment, with an effort to remove the bulk sealer from pulp chamber and all obtuation materials should be strictly localized in the area of the root canal in order to minimize the risk of discoloration. In addition, in the Van der Burgt study, the smear layer was removed post-instrumentation with EDTA and $\mathrm{NaOCl}$. This layer of debris occludes dentinal tubules, thus preventing sealer diffusing. Another explanation for the different results is that in our study the colour evaluation was recorded on the middle third of tooth crown. Kraus and Jordan ${ }^{(8)}$ stated that the pathway by which staining materials diffuse from the canal is through tubules; the stain potentially may then show in the cervical region, because enamel in the cervical region is thinner and the discolouration of underlying dentin is more pronounced.

AH-26, the sealer tested in this study, the color is seemed to be stable. However, the present results are in contrast to finding of Shahrami et al. ${ }^{(9)}$.

The quantitative results that used colorimetric device for color analysis which is used in this study may adversely influence the interpretation. These methods of color analysis with a device in addition visual method are the most currently methods which was used. The visual method is very difficult and it has interference variation such as eye fatigue and light position. (10) Instead that the device method which is used in our study is quantitative objective method but this technique has their own limitations such as limited access of clinician to such device. ${ }^{(11)}$

\section{CONCLUSIONS}

With the limitations of this study we conclude that the selection of endodontic sealers should be based mainly on other criteria (setting time, microleakage and toxicity) and not on the potent ionic staining.

\section{REFERENCES}

1. van der Burgt TP, Mullaney TP, Plasschaert AJ. Tooth discoloration induced by endodontic 
sealers. Oral Surg Oral Med Oral Pathol. 1986 Jan;61(1):84-9.

2. Nicholls E. Endodontics, ed 3, Bristol, 1976, John Wright and Sons Ltd. pp 339-340.

3. Plotino G, Grande NM, Pameijer $\mathrm{CH}$ and Somma F, Nonvital tooth bleaching: A review of the literature and clinical procedures.JOE.2008,34(4):394-407.

4. Van der Burgt TP, Plasschaert AJ. Tooth discoloration induced by dental materials. Oral Surg Oral Med Oral Pathol. 1985 Dec;60(6):666-9.

5. Gutierrez JH, Guzman M. Tooth discoloration in endodontic procedures. Oral Surg Oral Med Oral Pathol. 1968 Nov;26(5):706-11.

6. Parsons JR, Walton RE, Ricks-Williamson L. In vitro longitudinal assessment of coronal discoloration from endodontic sealers. J Endod. 2001 Nov;27(11):699-702.
7. Partovi M, Al-Havvaz AH and Soleimani B, In vitro computer analysis of crown discoloration from commonly used endodontic sealers. Aust Endod J 2006b: 32:116-119.

8. Kraus BS, Jordan RE. Dental anatomy and occlusion. $7^{\text {th }}$ ed. Baltimore, Wiliams and Wilkins, 1976:159.

9. Shahrami F, Zaree M, Mir APB. AbdollahiArmani M, Mesgarani A. Comparison of tooth crown discoloration with epiphany and AH26 sealer in term of chroma and value: an in vitro study. Braz J Oral Sci; 2011. 10(3): 171-174

10. Johnston WM, Kao EC. Assessment of appearance match by visual observation and clinical colorimetry. J Dent Res. 1989 May;68(5):819-22.

11. Lenherr P, Allgayer N, Weiger R, Filippi A, attin T\& Krasti G. Tooth discoloration induced by endodontic materials: a laboratory study. International Endodontic J. 2012:1-8. 